\title{
Disordered and Displacive Models of the Structure for the Normal Phase of $\left\{\mathrm{N}\left(\mathrm{CH}_{3}\right)_{4}\right\}_{2} \mathrm{MnCl}_{4}$
}

\author{
Naohiro KoshiJI and Hiroyuki MashiYama ${ }^{\dagger}$ \\ Kurume National College of Technology, Kurume, Fukuoka 830-8555 \\ ${ }^{\dagger}$ Department of Physics, Faculty of Science, Yamaguchi University, Yamaguchi 753-8512
}

(Received August 16, 2001)

\begin{abstract}
The crystal structure analysis for the normal phase of $\left\{\mathrm{N}\left(\mathrm{CH}_{3}\right)_{4}\right\}_{2} \mathrm{MnCl}_{4}$ has been performed for two models: a displacive model and a disordered model. In the former model, the constituent tetrahedra of tetramethylammonium and tetrachloromanganate satisfy a mirror symmetry themselves imposed by the crystal symmetry. In the latter model, each tetrahedron occupies two equivalent positions related by the mirror symmetry with an equal occupation probability: split atom method. A special attention has been paid to their thermal parameters; the former model exhibits an abnormal increase of the parameters near the transition temperature indicating a critical phenomenon. From the significant test for the $R$ factor, the latter model seems to be more appropriate than the former one. The disorderness of tetrahedra of tetramethylammonium is also confirmed by double peaks in Fourier synthesis map for carbon atoms. On the other hand, $\mathrm{MnCl}_{4}$ can be well represented both by ths displacive and the disordered models.
\end{abstract}

KEYWORDS: normal phase, structure analysis, displacive model, disordered model, split atom method

\section{$\S 1$. Introduction}

$T M A_{2} M X_{4}\left(T M A=\mathrm{N}\left(\mathrm{CH}_{3}\right)_{4} ; M=\mathrm{Zn}, \mathrm{Co}, \mathrm{Mn}\right.$, etc; $\left.X=\mathrm{Cl}, \mathrm{Br}\right)$ is a typical crystal system which performs a normal-incommensurate phase transition. ${ }^{1-3)}$ The space group of the normal phase is Pmcn $(Z=4)$, as same as in many incommensurate crystals with the common chemical formula

of $A_{2} B X_{4}{ }^{4)}$ In the $T M A_{2} M X_{4}$ family, successive transitions between commensurate phases have been widely interested, because the phases can be located on the unified pressure-temperature phase diagram. ${ }^{2,5)}$ A common mechanism is expected for the stabilization of commensurate and incommensurate phases. ${ }^{6)}$ Among the $T M A_{2} M X_{4}$ family, $\left\{\mathrm{N}\left(\mathrm{CH}_{3}\right)_{4}\right\}_{2} \mathrm{MnCl}_{4}$ is an end-point crystal since it is located in the lowest pressure position in the unified P-T phase diagram: under the hydrostatic pressure, a number of commensurate phases as well as the incommensurate phase can be realized. ${ }^{7,8)}$ The study on the structural origin inducing modulated structures will be worthy in $\left\{\mathrm{N}\left(\mathrm{CH}_{3}\right)_{4}\right\}_{2} \mathrm{MnCl}_{4}$. 
At atmospheric pressure, $\left\{\mathrm{N}\left(\mathrm{CH}_{3}\right)_{4}\right\}_{2} \mathrm{MnCl}_{4}$ was found to be incommensurate in a narrow temperature range between 291.5 and $\left.292.2 \mathrm{~K} .{ }^{3}\right)$ The modulated structure locks into a commensurate phase (phase III, monoclinic $P 2_{1} / c, Z=8$ ). The unit cell of phase III is doubled along the $c$-axis. A second lock-in transition takes place with the modulation wavenumber $q_{z}=\frac{1}{3}$ at $266 \mathrm{~K}$, below which and phase IV $\left(P 2_{1} / n, Z=12\right)$ is realized. Phase IV is followed by another normal phase (phase $\mathrm{V}, P 2_{1} / c, Z=4$ ) below $172 \mathrm{~K} .^{5}$ )

A detailed crystal analysis of the normal phase is a starting point to clarify the mechanism to stabilize the modulated structures. In our previous paper, we determined the crystal structures of phases I, III, IV and V by single crystal X-ray diffractometry. ${ }^{9)}$ In the normal phase I, the crystal consists of three sublattices constituted by $\mathrm{MnCl}_{4}$ named as $T C M$ and two tetramethylammoniums named as $T M A(1)$ and $T M A(2)$. It has been declared that each of these tetrahedra occupies disorderly two equivalent sites related by the mirror symmetry perpendicular to the $a$-axis. Such

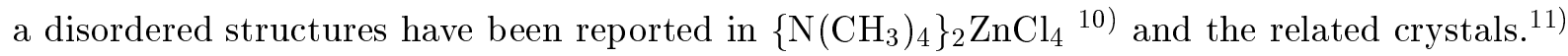

As for the crystal structure of phase I, the analysis has been performed at 293K, which is just above the transition temperature. ${ }^{9)}$ It has been concluded that each tetrahedron occupies either site so as to construct the superlattice structure. The center of mass of each tetrahedron was on the mirror position. Meanwhile, the center of mass deviates from the mirror plane in the modulated phase. If the modulated structure is an ordered structure of the disordered normal phase, then why the center of mass stays on the mirror plane in normal phase? In order to clarify the transition mechanism, it is necessary to declare whether the center of mass is on the mirror plane (displacive so far as the translation) or it is off the mirror plane (disordered for both the translational and librational modes) in the normal phase.

In order to clarify this problem we re-investigate the disorderness of the three kind of terahedra and the character of the translational motion of the center of mass. Diffraction data at seven different temperatures within the phase I are measured newly. Crystal structure are refined both for the disordered model (split-atom methods) and for the displacive model. The temperature variation of the thermal parameters is investigated carefully.

\section{§2. Experiment}

Single crystal of $\left\{\mathrm{N}\left(\mathrm{CH}_{3}\right)_{4}\right\}_{2} \mathrm{MnCl}_{4}$ were grown by slow evaporation method from an aqueous solution of stoichiometric mixture of $\mathrm{N}\left(\mathrm{CH}_{3}\right)_{4} \mathrm{Cl}$ and $\mathrm{MnCl}_{2}$ at about $300 \mathrm{~K}$. The crystal is light yellow in color, transparent and deliquescent. A spherical specimen with radius of $0.21 \mathrm{~mm}$ was prepared and mounted on a Weissenberg type camera with equipping of an imaging plate (MAC Science DIP-3000). Cold nitrogen gas was used to lower the temperature, which was controlled within $\pm 0.1 \mathrm{~K}$. Graphite-monochromated Mo $K \alpha$ radiation, generated by a rotating anode $(50 \mathrm{KV}$, $300 \mathrm{~mA})$, was used $(\lambda=0.71073 \AA)$. The diffraction was observed by oscillating crystal methods. A set of eighteen photographs were taken each $10^{\circ}$ of oscillation range of the specimen. The total 
exposure time for one set of data was only three hours. Since the crystal may be affected by radiation damage, ${ }^{12)}$ the rapid measurement is quite advantageous for the imaging plate system.

Using these photographs, all the reflections were indexed, space group was confirmed, and lattice parameters were refined. Intensity normalization was made for each photograph by using equivalent reflections. Intensity data for structure analysis were collected at seven different temperatures in the normal phase I with small temperature step near transition point. Namely, data collections were made at $340 \mathrm{~K}, 320 \mathrm{~K}, 300 \mathrm{~K}, 297 \mathrm{~K}, 295 \mathrm{~K}, 294 \mathrm{~K}$ and $293 \mathrm{~K}$,respectively. Absorption, Lorentz and polarization corrections were made. Absorption correction $(\mu \mathrm{R}=0.280)$ was done for sperical specimen. Only strong reflections $\left(F_{o}>5 \sigma\left(F_{o}\right)\right)$ were used for the structure analysis. All hydrogen atoms were neglected in the analysis. Atomic scattering factors were taken from International Tables for X-ray crystallography ${ }^{13)}$ and a typical crystal data is shown in Table I.

Table I. Crystal deta of $\left\{\mathrm{N}\left(\mathrm{CH}_{3}\right)_{4}\right\}_{2} \mathrm{MnCl}_{4}$ at $295 \mathrm{~K}$.

\begin{tabular}{lc}
\hline Phase & $\mathrm{I}$ \\
Space group & Pmcn \\
$Z$ & 4 \\
$a(\AA)$ & $9.070(1)$ \\
$b(\AA)$ & $15.636(2)$ \\
$c(\AA)$ & $12.345(2)$ \\
Index range & $0 \leq h \leq 11$ \\
& $0 \leq k \leq 22$ \\
& $0 \leq l \leq 17$ \\
Number of independent reflections & 1764 \\
$\left(F_{o}>5 \sigma\left(F_{o}\right)\right)$ & \\
Absorption coefficient $\left(m m^{-1}\right)$ & 1.32 \\
\hline
\end{tabular}

The low temperature phase is characterized by the appearance of superlattice reflections of $(h$ $\left.k l \pm \frac{1}{2}\right)$. In order to obtain the transition temperature, the diffuse intensity near $\left(\begin{array}{lll}3 & 1 & \frac{1}{2}\end{array}\right)$ was measured by the use of 4-circle diffractometer. Figure 1 shows the temperature dependence of the diffuse intensity. The prepared specimen did not indicate the appearance of the intermediate incommensurate phase; the normal phase I transformed directly into the superlattice phase at $293 \mathrm{~K}$. It should be noted here that the incommensurate phase II is more stabilized under hydrostatic pressure, and that the phase II is sometimes missing under atmospheric pressure.

Program AXS89 system ${ }^{14)}$ was used for the refinement of the crystal structure, and calculations were made on a personal computer. In the block-diagonal least-squares calculations, $w R^{2}=\sum w(\mid$ $\left.F_{c}|-| F_{0} \mid\right)^{2} / \sum w\left|F_{0}\right|^{2}$ was minimized with equal weight $(w=1)$. 


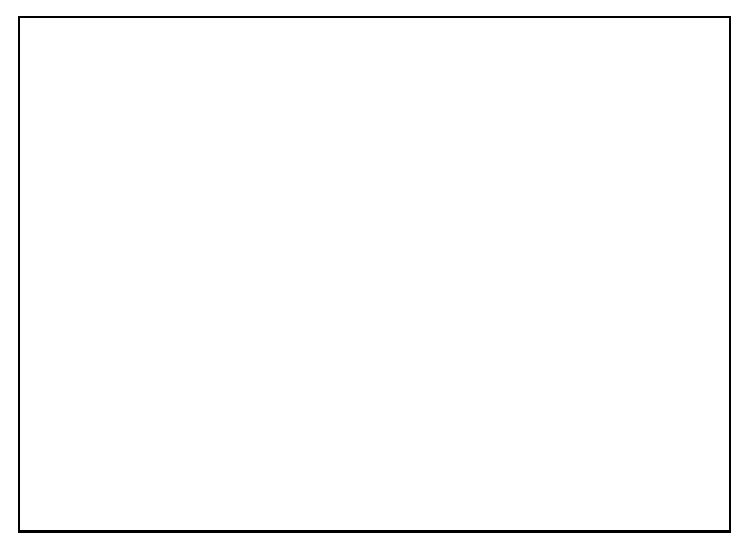

Fig. 1. Temperature dependence of the diffuse intensity at $\left(\begin{array}{lll}3 & 1 & \zeta\end{array}\right)$.

\section{$\S 3 . \quad$ Models and Analysis}

\subsection{Displacive model}

In a displacive model, each tetrahedron sits on a mirror plane: $\mathrm{x}=1 / 4,3 / 4$. As for starting parameters, we used our previous result at $293 \mathrm{~K} .{ }^{9)}$ Least-squares calculations were converged at $\mathrm{R}=0.0699 \sim 0.0812$ for seven different temperatures where the residual is defined as

$$
R=\sum\left\|F_{c}|-| F_{o}\right\| / \sum\left|F_{o}\right|
$$

The typical final atomic parameters at $295 \mathrm{~K}$ are listed in Table II. Bond lengths and angles are given in Table III. The tetrahedra of $T C M$ and $T M A(1)$ are regular, however, $T M A(2)$ is distorted a little. Projection of the structure viewed from the $c$-direction is shown in Figure 2. The anisotropic thermal parameters are demonstrated by ellipsoids (ORTEP-III ${ }^{15)}$ was employed).

The structure is characterized by pseudo-hexagonal networks of six-member rings of $T M A(1)$ and $T C M$ at $n$-glide plane. The tetrahedra $T M A(1)$ and $T M A(2)$ are surrounded by 5 and 6 $T C M$ 's, respectively. The carbon atoms of $T M A(1)$ and $T M A(2)$ can be linked by 14 chlorine atoms in the distance of 3.565 4.346 Aand $10 \mathrm{Cl}$ 's in $3.409 \sim 3.925 \AA$, respectively. These links correspond to 11 and 9 configuration numbers of oxygen surrounding $K(1)$ and $K(2)$, respectively, in $\beta-\mathrm{K}_{2} \mathrm{SO}_{4}$ structure. ${ }^{16)}$ The mean distances between $\mathrm{C}$ and $\mathrm{Cl}$ are 3.958 Åand $3.733 \AA$ Afor $T M A(1)$ and $T M A(2)$, respectively.

The ellipsoids in Fig. 2 indicate oscillations of $T C M$ and $T M A(1)$ about an axis nearly parallel to the $c$-axis, while $T M A(2)$ is oscillating about $\mathrm{N}(2)-\mathrm{C}(5)$ bond. In order to discuss the oscillation of $T C M$, let's take notice of $\mathrm{Cl}(2)$ on the mirror plane. The temperature dependence of thermal 
Table II. Final atomic coordinates and equivalent isotropic thermal parameters for the displacive model at $295 \mathrm{~K}$ in phase I of $\left\{\mathrm{N}\left(\mathrm{CH}_{3}\right)_{4}\right\}_{2} \mathrm{MnCl}_{4}$. The e.s.d.'s are given in parentheses; * denotes the fixed parameters.

\begin{tabular}{ccccc}
\hline & $x$ & $y$ & $z$ & $B_{\text {eq }}\left(\AA^{2}\right)$ \\
\hline $\mathrm{Mn}$ & $0.25\left(^{*}\right)$ & $0.4074(1)$ & $0.2456(1)$ & $3.82(4)$ \\
$\mathrm{Cl}(1)$ & $0.4069(2)$ & $0.0562(2)$ & $7.63(8)$ \\
$\mathrm{Cl}(2)$ & $0.5457(2)$ & $0.3175(3)$ & $10.33(12)$ \\
$\mathrm{Cl}(3)$ & $0.3367(2)$ & $0.3074(2)$ & $9.06(6)$ \\
$\mathrm{N}(1)$ & $0.25\left(^{*}\right)$ & $0.0961(4)$ & $0.1490(5)$ & $4.75(17)$ \\
$\mathrm{N}(2)$ & $0.0381(2)$ & $0.3256(4)$ & $0.0081(6)$ & $4.57(16)$ \\
$\mathrm{C}(1)$ & $0.0993(11)$ & $0.2675(9)$ & $14.23(71)$ \\
$\mathrm{C}(2)$ & $0.25\left(^{*}\right)$ & $0.1415(9)$ & $0.1088(12)$ & $15.33(77)$ \\
$\mathrm{C}(3)$ & $0.2374(7)$ & $0.1054(10)$ & $19.75(49)$ \\
$\mathrm{C}(5)$ & $0.3885(12)$ & $0.0510(13)$ & $21.64(47)$ \\
$\mathrm{C}(6)$ & $0.25\left(^{*}\right)$ & $0.0901(14)$ & $20.79(45)$ \\
$\mathrm{C}(7)$ & $0.1243(13)$ & $-0.0493(2)$ & \\
\hline
\end{tabular}

Table III. Bond lengths $(\AA)$ and angles $\left({ }^{\circ}\right)$ at $295 \mathrm{~K}$ in phase I for the displacive model of $\left\{\mathrm{N}_{(}\left(\mathrm{CH}_{3}\right)_{4}\right\}_{2} \mathrm{MnCl}_{4}$.

\begin{tabular}{ccc}
\hline $\mathrm{Mn}-\mathrm{Cl}(1) 2.339(3)$ & $\mathrm{N}(1)-\mathrm{C}(1) 1.464(13)$ & $\mathrm{N}(2)-\mathrm{C}(5) 1.477(15)$ \\
$\mathrm{Mn}-\mathrm{Cl}(2) 2.338(13)$ & $\mathrm{N}(1)-\mathrm{C}(2) 1.450(16)$ & $\mathrm{N}(2)-\mathrm{C}(6) 1.411(22)$ \\
$\mathrm{Mn}-\mathrm{Cl}(3) 2.344(14)$ & $\mathrm{N}(1)-\mathrm{C}(3) 1.446(16)$ & $\mathrm{N}(2)-\mathrm{C}(7) 1.384(15)$ \\
$\mathrm{Cl}(1)-\mathrm{Mn}-\mathrm{Cl}(2) 112.5(7)$ & $\mathrm{C}(1)-\mathrm{N}(1)-\mathrm{C}(2) 111.9(9)$ & $\mathrm{C}(5)-\mathrm{N}(2)-\mathrm{C}(6) 113.2(6)$ \\
$\mathrm{Cl}(1)-\mathrm{Mn}-\mathrm{Cl}(3) 108.9(7)$ & $\mathrm{C}(1)-\mathrm{N}(1)-\mathrm{C}(3) 110.8(8)$ & $\mathrm{C}(5)-\mathrm{N}(2)-\mathrm{C}(7) 110.7(7)$ \\
$\mathrm{Cl}(2)-\mathrm{Mn}-\mathrm{Cl}(3) 108.2(6)$ & $\mathrm{C}(2)-\mathrm{N}(1)-\mathrm{C}(3) 109.5(7)$ & $\mathrm{C}(6)-\mathrm{N}(2)-\mathrm{C}(7) 103.9(6)$ \\
\hline
\end{tabular}

parameters is plotted in Figure 3. The mean squared displacement $\mathrm{U}_{11}$ along the $a$-axis increases with approaching the transition temperature, though $\mathrm{U}_{22}$ and $\mathrm{U}_{33}$ indicate the regular temperature dependence of

$$
U \propto k_{B} T
$$

Such a critical increase(the deviation from the linear temperature dependence of eq.(3.2)) can be also recognized in $\mathrm{U}_{11}$ of $\mathrm{Cl}(1), \mathrm{U}_{11}$ and $\mathrm{U}_{22}$ of $\mathrm{Cl}(3), \mathrm{U}_{11}$ of $\mathrm{C}(1)$ and $\mathrm{C}(2), \mathrm{U}_{11}$ and $\mathrm{U}_{22}$ of $\mathrm{C}(3), \mathrm{U}_{11}$ 
of $\mathrm{C}(6)$, and $\mathrm{U}_{33}$ of $\mathrm{C}(7)$. These displacements correspond to the above mentioned oscillations of $T C M, T M A(1)$ and $T M A(2)$ tetrahedra. Thus the displacive model indicates the critical slowing down of constituent tetrahedra at the stability limit of the normal phase.

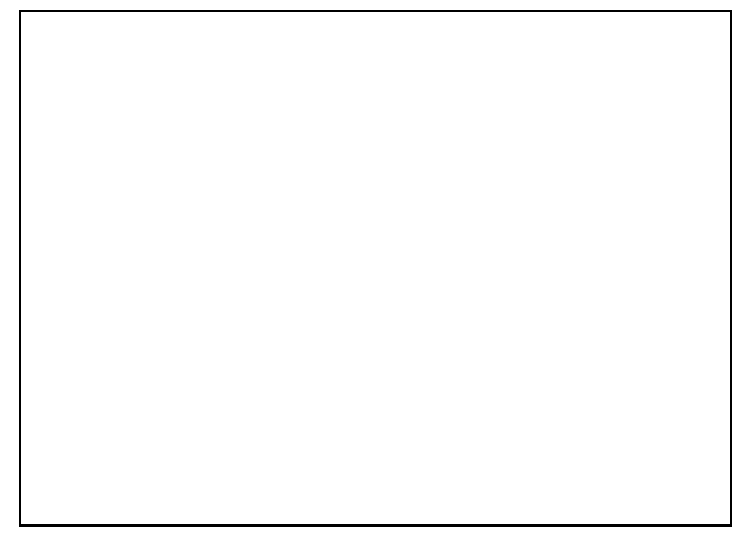

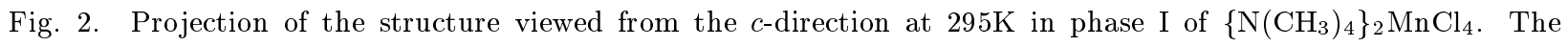
anisotropic thermal parameters are represented by ellipsoids. The atoms within half of a unit cell $\left(0 \leq z \leq \frac{1}{2}\right)$ are drawn.

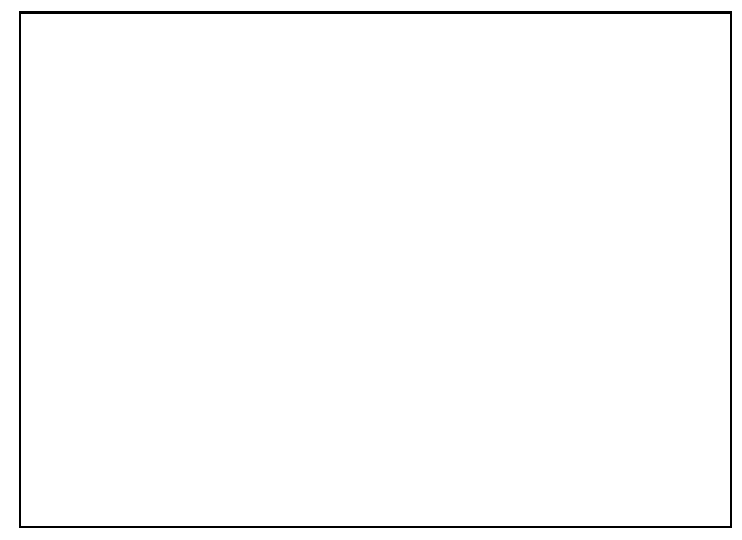

Fig. 3. Temperature variation of the thermal parameters $U_{i i}$. Those data for low- temperature phases below $293 \mathrm{~K}$ are quoted from our previous report. Broken straight lines are guides to the eyes and are plotted from the origin. 


\subsection{Disordered model}

Another model is assumed, in which each tetrahedron occupies two configurations with an equal probability, and that the diffraction intensity can be represented by split-atom method. We firstly put the center of mass on the mirror plane; $\mathrm{Mn}, \mathrm{N}(1)$ and $\mathrm{N}(2)$ are fixed on the mirror plane perpendicular to the $a$-axis (model D-1). Starting from the previous results at 293K, all refinements at seven different temperatures converged at $R=0.0563 \sim 0.0680(0.0591$ at $295 \mathrm{~K})$. The results are essentially equivalent to the previous one.

Secondly, we removed the constraint for fixing $\mathrm{Mn}, \mathrm{N}(1)$ and $\mathrm{N}(2)$ from the mirror plane (model D-2). The least-squares calculations were converged at $R=0.0555 \sim 0.0675(0.0587$ at $295 \mathrm{~K})$. The typical final atomic parameters for $295 \mathrm{~K}$ are listed in Table IV. The thermal parameters for Cl's

Table IV. Atomic parameters for disordered model at $295 \mathrm{~K}$ in phase I of $\left\{\mathrm{N}\left(\mathrm{CH}_{3}\right)_{4}\right\}_{2} \mathrm{MnCl}_{4}$.

\begin{tabular}{|c|c|c|c|c|}
\hline & $x$ & $y$ & $z$ & $B_{e q}\left(\AA^{2}\right)$ \\
\hline $\mathrm{Mn}$ & $0.2574(4)$ & $0.4074(1)$ & $0.2456(1)$ & $3.69(2)$ \\
\hline $\mathrm{Cl}(1)$ & $0.2699(4)$ & $0.4069(2)$ & $0.0566(2)$ & $6.67(7)$ \\
\hline $\mathrm{Cl}(2)$ & $0.2851(4)$ & $0.5455(1)$ & $0.3171(2)$ & $7.17(11)$ \\
\hline $\mathrm{Cl}(3)$ & $0.0268(3)$ & $0.3517(2)$ & $0.3085(3)$ & $7.29(9)$ \\
\hline $\mathrm{Cl}(4)$ & $0.4492(4)$ & $0.3229(2)$ & $0.3063(3)$ & $7.10(8)$ \\
\hline $\mathrm{N}(1)$ & $0.2422(22)$ & $0.0965(4)$ & $0.1488(5)$ & $4.66(14)$ \\
\hline $\mathrm{N}(2)$ & $0.7575(23)$ & $0.3256(3)$ & $0.0080(5)$ & $4.46(14)$ \\
\hline $\mathrm{C}(1)$ & $0.2176(29)$ & $0.0995(9)$ & $0.2665(8)$ & $11.92(61)$ \\
\hline $\mathrm{C}(2)$ & $0.2063(20)$ & $0.0095(7)$ & $0.1088(11)$ & $10.24(64)$ \\
\hline $\mathrm{C}(3)$ & $0.1560(19)$ & $0.1616(9)$ & $0.0959(13)$ & $12.39(57)$ \\
\hline $\mathrm{C}(4)$ & $0.4058(16)$ & $0.1133(14)$ & $0.1156(17)$ & $14.41(75)$ \\
\hline $\mathrm{C}(5)$ & $0.7714(23)$ & $0.2371(6)$ & $0.0517(11)$ & $11.45(40)$ \\
\hline $\mathrm{C}(6)$ & $0.6886(19)$ & $0.3854(10)$ & $0.0872(11)$ & $12.78(57)$ \\
\hline $\mathrm{C}(7)$ & $0.6607(19)$ & $0.3242(11)$ & $-0.0877(12)$ & $14.51(58)$ \\
\hline $\mathrm{C}(8)$ & $0.9015(13)$ & $0.3550(8)$ & $-0.0180(13)$ & $8.53(42)$ \\
\hline
\end{tabular}

and C's become smaller than the displacive model. Bond length and angles are given in Table V. The tetrahedra oh $T C M$ and $T M A(1)$ are distorted a little in comparison with the displacive model given in Table III. Projection of the structure viewed from the $c$-direction are shown in Fig. 4. The distance between a carbon atom of $T M A(2)$ and a chlorine atom of $T C M$ is shorter than that of $T M A(1)$ to $T C M$, in general, as same as the displacive model. Some distances are too short in the disordered model. However, such short links are avoided in the ordered structure at 
low temperature as shown in Fig. 6 of ref. ${ }^{9)}$

Table V. Bond lengths $(\AA)$ and angles $\left(^{\circ}\right)$ at $295 \mathrm{~K}$ in phase I for the disordered model at $295 \mathrm{~K}$ in phase $\mathrm{I}$ of $\left\{\mathrm{N}\left(\mathrm{CH}_{3}\right)_{4}\right\}_{2} \mathrm{MnCl}_{4}$.

\begin{tabular}{ccc}
\hline $\mathrm{Mn}-\mathrm{Cl}(1) 2.337(2)$ & $\mathrm{N}(1)-\mathrm{C}(1) 1.471(12)$ & $\mathrm{N}(2)-\mathrm{C}(5) 1.491(12)$ \\
$\mathrm{Mn}-\mathrm{Cl}(2) 2.346(3)$ & $\mathrm{N}(1)-\mathrm{C}(2) 1.484(13)$ & $\mathrm{N}(2)-\mathrm{C}(6) 1.489(18)$ \\
$\mathrm{Mn}-\mathrm{Cl}(3) 2.395(4)$ & $\mathrm{N}(1)-\mathrm{C}(3) 1.440(20)$ & $\mathrm{N}(2)-\mathrm{C}(7) 1.472(21)$ \\
$\mathrm{Mn}-\mathrm{Cl}(4) 2.309(4)$ & $\mathrm{N}(1)-\mathrm{C}(4) 1.562(24)$ & $\mathrm{N}(2)-\mathrm{C}(8) 1.421(23)$ \\
$\mathrm{Cl}(1)-\mathrm{Mn}-\mathrm{Cl}(2) 111.9(2)$ & $\mathrm{C}(1)-\mathrm{N}(1)-\mathrm{C}(2) 108.9(15)$ & $\mathrm{C}(5)-\mathrm{N}(2)-\mathrm{C}(6) 112.4(18)$ \\
$\mathrm{Cl}(1)-\mathrm{Mn}-\mathrm{Cl}(3) 111.4(3)$ & $\mathrm{C}(1)-\mathrm{N}(1)-\mathrm{C}(3) 110.1(19)$ & $\mathrm{C}(5)-\mathrm{N}(2)-\mathrm{C}(7) 109.1(19)$ \\
$\mathrm{Cl}(1)-\mathrm{Mn}-\mathrm{Cl}(4) 106.6(2)$ & $\mathrm{C}(1)-\mathrm{N}(1)-\mathrm{C}(4) 113.5(24)$ & $\mathrm{C}(5)-\mathrm{N}(2)-\mathrm{C}(8) 107.7(19)$ \\
$\mathrm{Cl}(2)-\mathrm{Mn}-\mathrm{Cl}(3) 107.8(2)$ & $\mathrm{C}(2)-\mathrm{N}(1)-\mathrm{C}(3) 112.2(19)$ & $\mathrm{C}(6)-\mathrm{N}(2)-\mathrm{C}(7) 106.6(19)$ \\
$\mathrm{Cl}(2)-\mathrm{Mn}-\mathrm{Cl}(4) 108.9(2)$ & $\mathrm{C}(2)-\mathrm{N}(1)-\mathrm{C}(4) 106.0(20)$ & $\mathrm{C}(6)-\mathrm{N}(2)-\mathrm{C}(8) 109.3(21)$ \\
$\mathrm{Cl}(3)-\mathrm{Mn}-\mathrm{Cl}(4) 110.2(3)$ & $\mathrm{C}(3)-\mathrm{N}(1)-\mathrm{C}(4) 106.2(22)$ & $\mathrm{C}(7)-\mathrm{N}(2)-\mathrm{C}(8) 111.8(23)$ \\
\hline
\end{tabular}

The rotation of tetrahedra from the mirror plane is almost similar between the model D-1 and D-2. In the model D-2, the center of mass the tetrahedra (the position of $\mathrm{Mn}, \mathrm{N}(1), \mathrm{N}(2)$ ) displaces a little from the mirror plane. However the deviation is small and temperature independent as shown in fig.5. In the disordered model, the atomic positions are within the thermal ellipsoids of the displacive model. The thermal parameters are still rather large, so that the thermal ellipsoids overlaps with the counterpart of the disordered atom. This is the reason why the structure can be analyzed by the displacive model as well.

The temperature dependence of thermal parameters (mean square displacements) of $\mathrm{Cl}(2)$ are plotted in Fig. 6. Although $\mathrm{U}_{11}$ does not show a critical behavior, it deviates the broken line extrapolated from the origin. While $\mathrm{U}_{22}$ and $\mathrm{U}_{33}$ obey the proportional dependence on temperature.

Here we note that the tilt angle of the $T C M$ tetrahedron changes with temperature. Figure 5 shows the displacement of $\mathrm{Cl}(2)$ from mirror plane, which increases with decreasing temperature. This change is reflected on abnormal increase in $\mathrm{U}_{11}$ of $\mathrm{Cl}(2)$ for the displacive model (Fig.3)

\section{$\S 4$. Discussion}

The normal phase of $\left\{\mathrm{N}\left(\mathrm{CH}_{3}\right)_{4}\right\}_{2} \mathrm{MnCl}_{4}$ is analyzed for both the displacive and disordered models. To describe the disordered model, the split-atom method is employed. In our previous report, the structure was analyzed at only one temperature, just above the transition temperature. Now we 


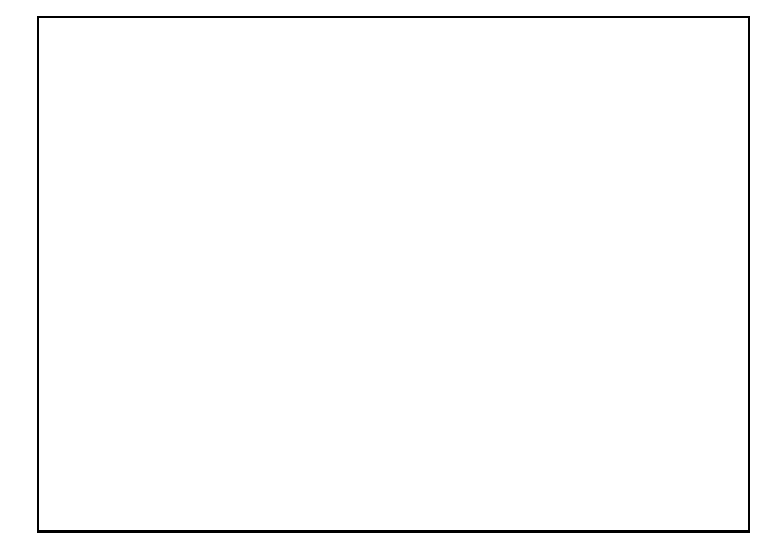

Fig. 4. Projection of the structure viewed from the $c$-direction for the disordered model at $295 \mathrm{~K}$ in phase $\mathrm{I}$ of $\left\{\mathrm{N}\left(\mathrm{CH}_{3}\right)_{4}\right\}_{2} \mathrm{MnCl}_{4}$. One of the two configurations for mirror symmetry within half of a unit cell $\left(0 \leq z \leq \frac{1}{2}\right)$ is drawn

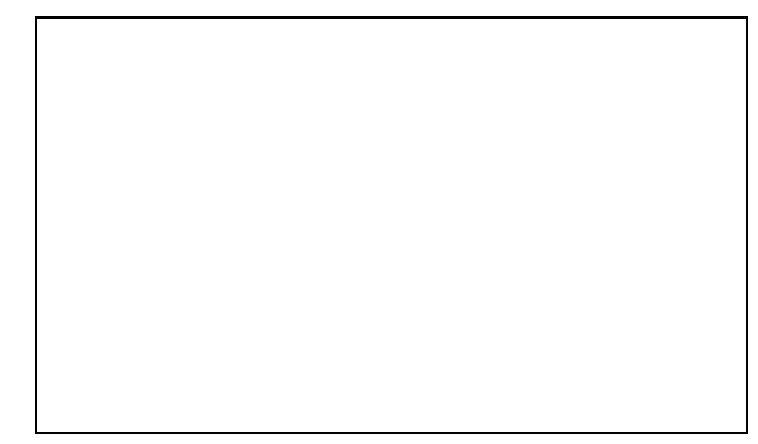

Fig. 5. Temperature dependence for displacements of the constituting tetrahedrons.

have refined at different seven temperatures up to $340 \mathrm{~K}$; the results are in agreement with the previous one essentially.

We have paid attention to the precursor change of the structure just above the transition temperature. The renewed analysis revealed the following results. (i) The thermal parameters for the disordered model do not show any precursor behavior above the transition temperature, as shown in Fig. 6. (ii) The root mean squared displacement (the thermal parameter $U_{11}$ of $\mathrm{Cl}(2)$ ) demonstrates a critical increase at the phase transition in the displacive model, as shown in Fig. 2 


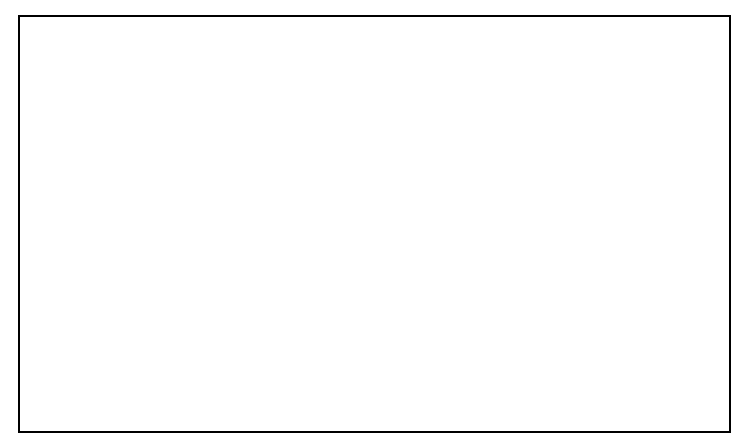

Fig. 6. Temperature dependence of the thermal parameters.

According to the disordered model, each tetrahedron occupies two sites randomly. Within each site, all constituent atoms perform thermal vibration. The shape of the potential function for each atom would not change much with temperature. Therefore, the thermal parameters for the disordered model would not show any critical increment. Only the flip motion between the two minimum of the potential becomes slowly and the flip motion of neighboring tetrahedrons would be more correlated as the temperature approaches to the critical one. This effect will be demonstrated by the increase of the diffuse scattering intensity. However, the diffuse intensity is not taken into account for the least-square refinement of the integrated intensity of the structure analysis. Thus no precursor effect is expected in the disordered model. The atomic coordinates of some atoms (eg. the $x$ parameter of $\mathrm{Cl}(2)$ ) changes appreciably with decreasing temperature. But the behavior seems to be continuous through the transition. When the flipping motion of $T C M$ becomes slowly, the surrounding $T M A(1)$ and $T M A(2)$ will follow this motion. Then the local configuration becomes as if it is the low-temperature phase, which has an enhanced deviation of the $x$ parameter.

It should be noted that we can explain the observed results by the displacive model as the structural model for the phase I. According to the displacive model, the equilibrium positions of Mn, $\mathrm{Cl}(1), \mathrm{Cl}(2), \mathrm{N}(1)$ and so on are on the mirror plane. The atoms vibrate thermally. With decreasing temperature, the thermal motion decreases a little. But a lattice vibrational mode becomes soft, and the amplitude increases since the restoring force weakens with approaching the transition temperature. Thus the thermal parameter $U_{11}$ becomes maximum at the transition temperature, as shown in Fig. 3. The enhancement of diffuse scattering is attributed to the precursor cluster in which the coherent lattice vibration shows a temporal low-temperature pattern.

Although both displacive and disordered models give a consistent explanation of the diffuse scattering and the Bragg reflection intensity as well as the convergence of the least-squared calculations 
for structure model, he significance test for the $R$-factor supports the disordered model. At $295 \mathrm{~K}$, $R$ 's are 0.0745 and 0.0587 for the displacive and disordered models, respectively. The difference is significant enough to support the split-atom model than the displace model in the level of 99.5

Here we discuss about the Fourier synthesis for electron density. Two atoms $\mathrm{Cl}(2)$ and $\mathrm{C}(6)$, which have large separate positions in the split-atom method, are selected. The electron density map at $\mathrm{Cl}(2)$ is shown in Fig. 7(a), where cross marks indicate the atomic positions in the splitatom method. The electron density has a regular elongated ellipsoid; no split of atom is clear. Even if the $\mathrm{Cl}(2)$ atom is in disorder, two split positions are near each other, the superposed electron density may be a single peak. From this electron map, the disorderness of the $T C M$ tetrahedron is not easily convinced.

Fig. 7. Electron density form Fourier synthesis. (a) $\mathrm{Cl}(2)$ and $(\mathrm{b}) \mathrm{C}(6)$ are drawn. The contour is $1\left[\mathrm{e} / \AA^{3}\right]$ and 0.5 $\left[\mathrm{e} / \AA^{3}\right]$ for (a) and (b), respectively. The negative density is indicated by hatching.

On the other hand, double peaks can be recognized in the Fourier map for $\mathrm{C}(6)$ as shown in Fig. 7(b). The carbon atoms of $T M A(1)$ also show split peaks in their electron density. Therefore, the rotational disorder of $T M A(1)$ and $T M A(2)$ tetrahedra is confirmed directly.

Now we can propose two structure model for $T C M$ in the normal phase of $(T M A)_{2} T C M$.

(i) The $T C M$ tetrahedron is also in disorder: each chlorine atom occupies two positions with an equal probability.

(ii)The $T C M$ tetrahedron vibrates un-harmocically; the thermal motion can be rather represented by the split-atom method: a displacive model with extremely un-harmonic vibration.

It is hard to distinguish above model from our present analysis. Moreover, a temperature variation takes place in split atom positions for chlorine and carbon atoms as shown in Fig. 5. In an ideal disordered model, two or more equivalent positions of atoms are given definitely. No change of the 
minimum positions for the disordered atoms is assumed. But Fig. 5 indicate a continuous change of positions with approaching the transition temperature. Though $\mathrm{C}(2)$ approaches the mirror plane, the $\mathrm{Cl}(2)$ atom moves off the mirror plane,; which is attributed to the abnormal increase of $U_{11}$ of $\mathrm{Cl}(2)$ for the displacive model discussed in Fig. 3.

Finally we note about the translational displacement off the mirror plane. In the simple disordered model, three kind of tetrahedra $T C M, T M A(1)$ and $T M A(2)$ are rotationally disorderd. Their center of mass is on the mirror plane. But the difference between the fully disordered model (Mn, $\mathrm{Cl}(1), \mathrm{Cl}(2), \mathrm{N}(1)$ and so on are off the mirror plane) and the model that the center of mass of tetrahedrons are on the mirror plane is little in $R$. (For example at 295K, $R$ 's are 0.0587 and 0.0591 for the former and the latter cases, respectively) Although the fully disordered model sounds to be a consistent model, the deviation from the mirror plane is tiny. Therefore it is desired to investigate the modulated phase more carefully to declare which model is more consistent throughout the phase transition. This point will be investigated in the separated paper near future.

\section{Acknowledgements}

The authors are grateful for fruitful discussions to Prof. K. Hasebe and technical comments to

Mr. H. Kasano, in the early stage of the study. The data collection was performed by the use of the Weissenberg camera (DIP 3000) at the Center of Instrumental Analysis, Yamaguchi University.

1) S. Sawada, Y. Shiroishi, A. Yamamoto, M. Takashige and M. Matsuo: J. Phys. Soc. Jpn. 44 (1978) 687.

2) H. Shimizu, N. Abe, N. Yasuda, S. Fujimoto, S. Sawada and Y. Shiroishi: Jpn. J. Appl. Phys. 18 (1979) 857.

3) H. Mashiyama and S. Tanisaki: J. Phys. Soc. Jpn. 50 (1981) 1413.

4) H. Z. Cummins: Phys. Rep. 185 (1990) 211.

5) K. Gesi: J. Phys. Soc. Jpn. 53 (1984) 627.

6) H. Mashiyama: J. Phys. Soc. Jpn. 49 (1980) 2270.

7) S. Shimomura,N. Hamaya,Y. Fujii: J. Phys. Soc. Jpn. 65 (1996) 661.

8) D. G. Sannikov,G. A. Kessenikh,H. Mashiyama: J. Phys. Soc. Jpn. 69 (2000) 130.

9) H. Mashiyama and N. Koshiji: Acta Crystallogr. B 45 (1989) 467.

10) K. Hasebe, H. Mashiyama, N. Koshiji and S. Tanisaki: J. Phys. Soc. Jpn. 56 (1987) 3543.

11) H. Kasano and H. Mashiyama: J. Phys. Soc. Jpn. 58 (1989) 3175.

12) H. Kasatani, H. Mashiyama and N. Koshiji: J. Phys. Soc. Jpn. 55 (1986) 4572.

13) IUC:International Tables for X-ray Crystallography. (Kynoch Press,1974, Birmingham) Vol.IV.

14) H. Mashiyama: J.Phys. Soc. Jpn. 60 (1991) 180.

15) M. N. Burnett and C. K. Johnson: ORTEP-III Oak Ridge Nat. Lab. (1996).

16) K. Sasvari: Acta Crystallogr. 16 (1963) 719.

17) W. C. Hamilton: Acta Crystallogr. 18 (1965) 502. 


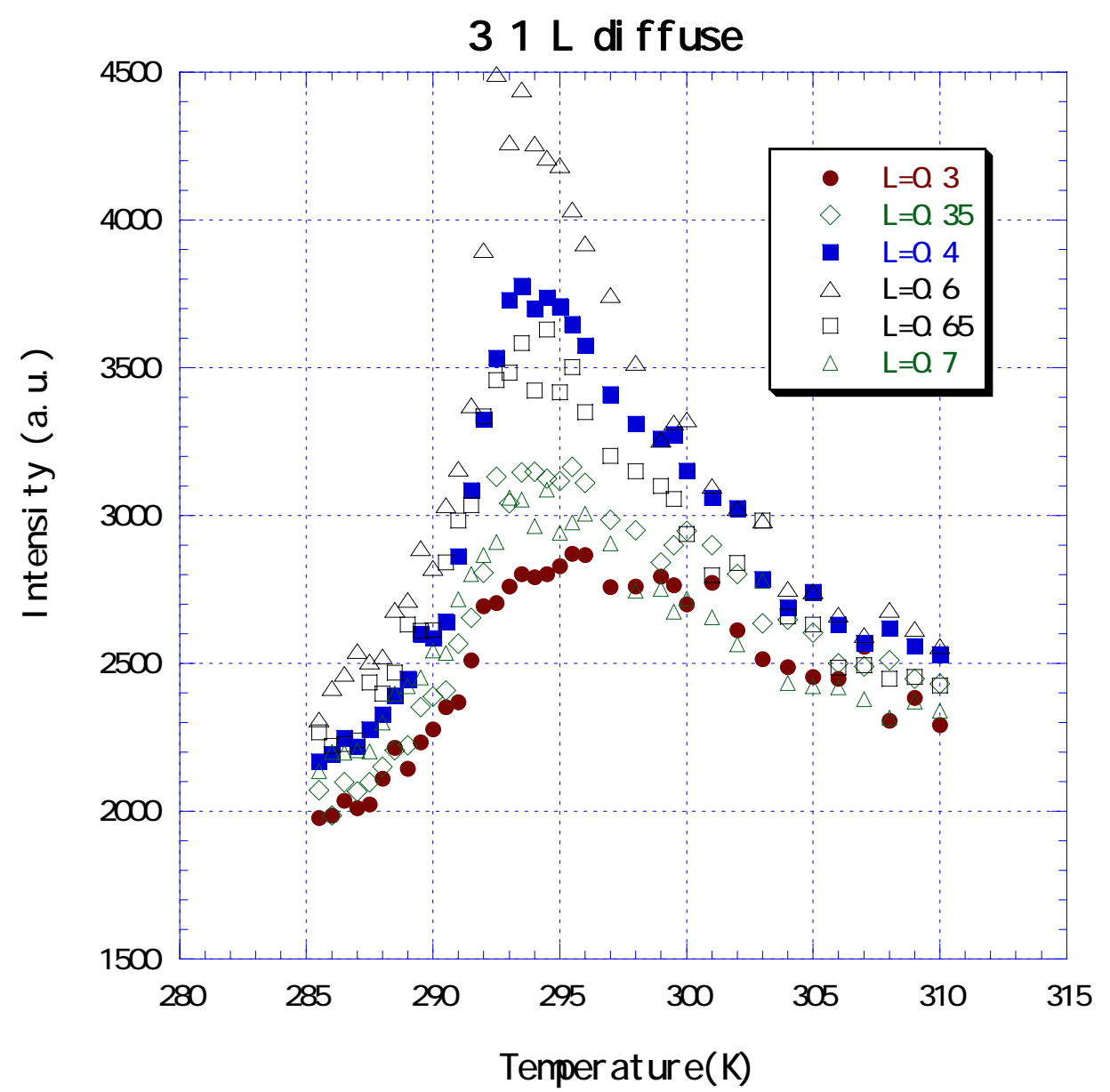

Fig. 1.

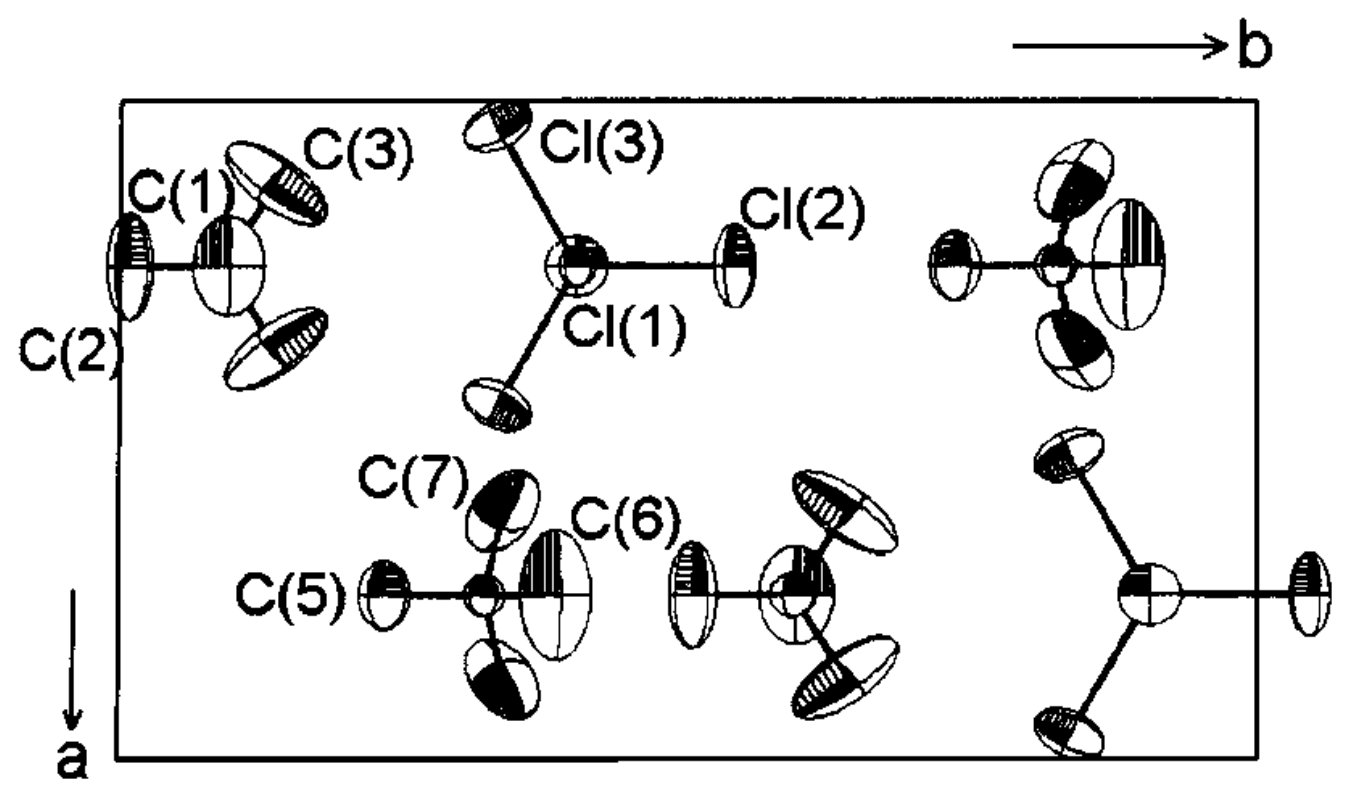

Fig. 2. 


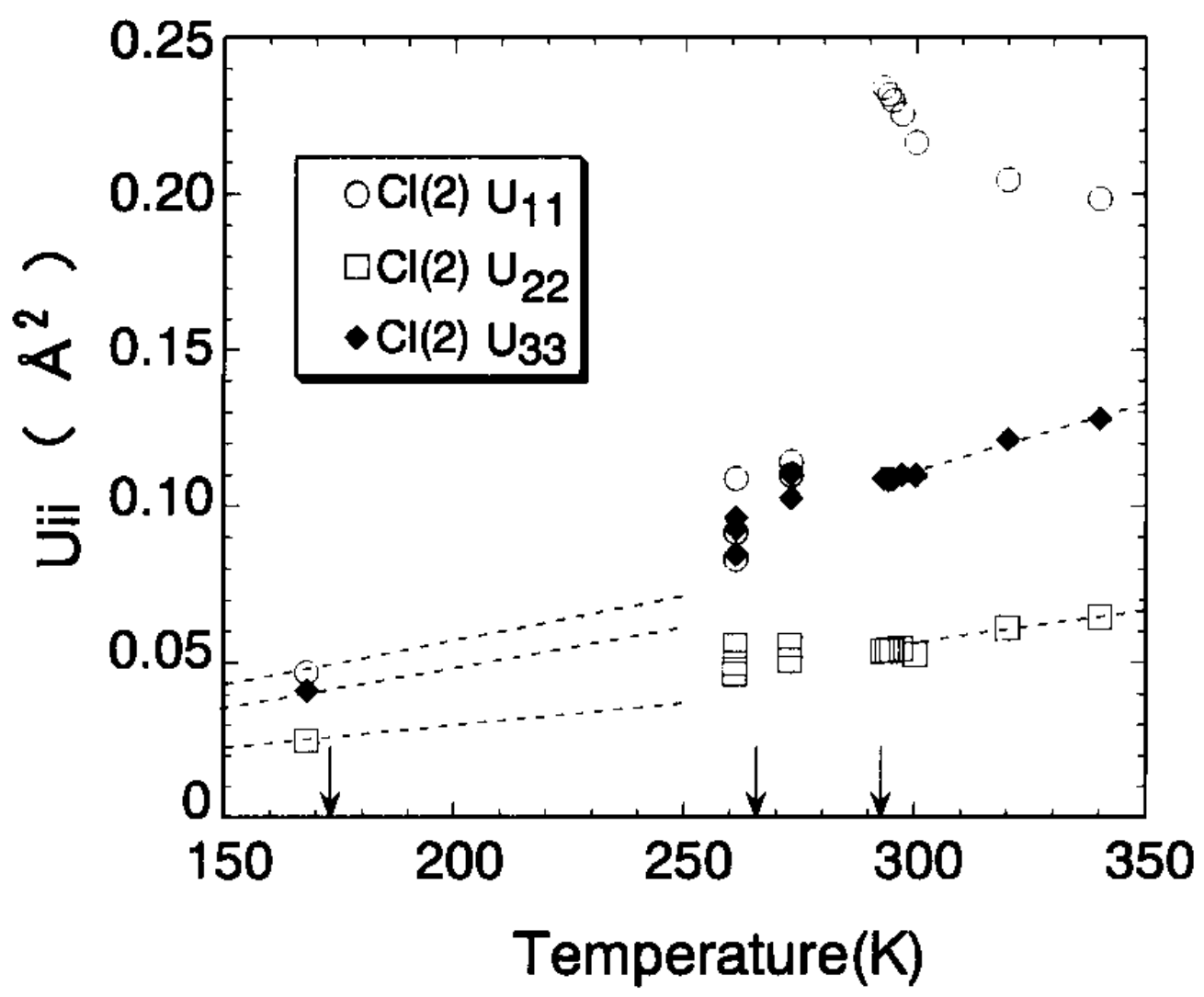

Fig. 3.

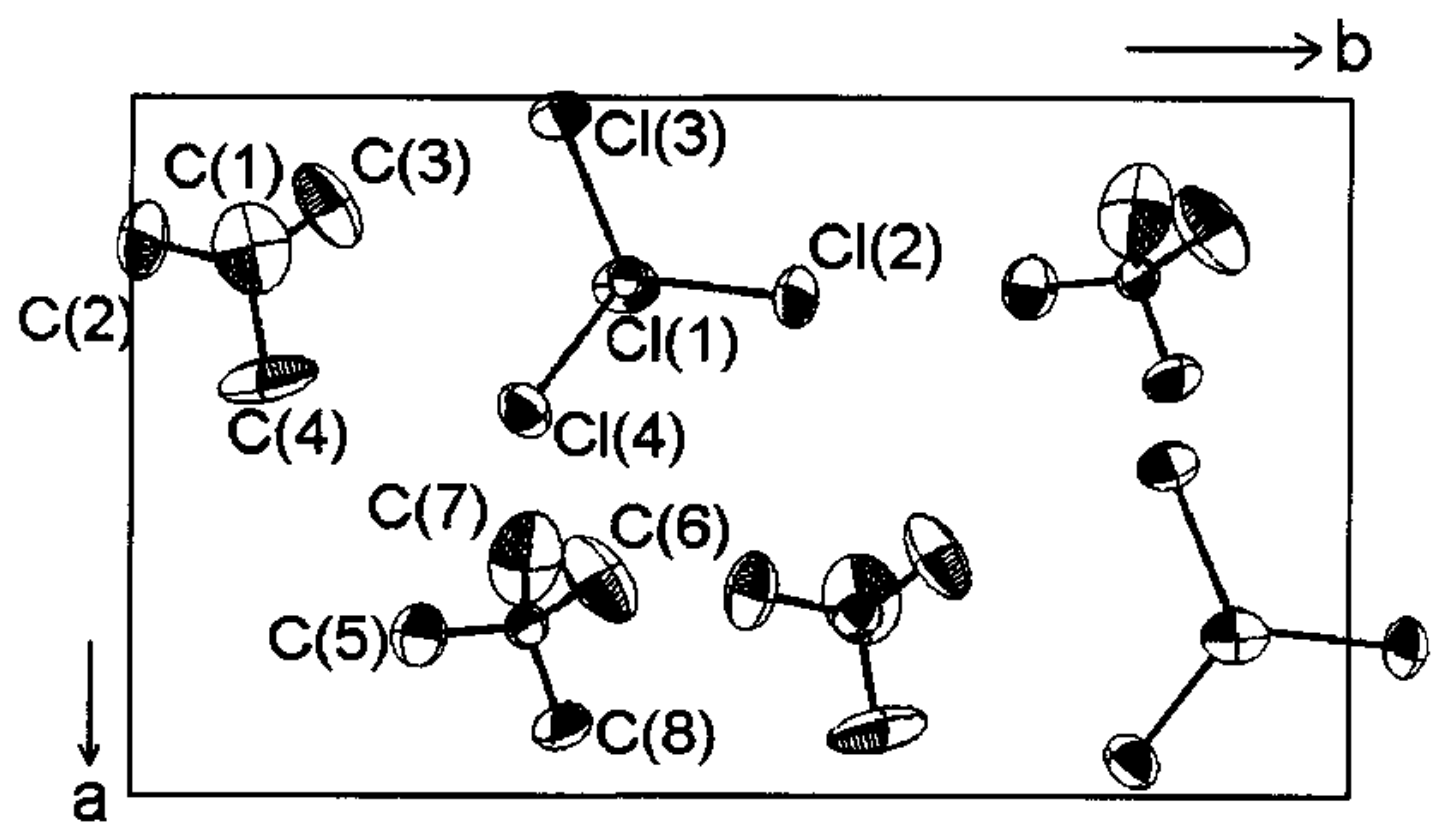

Fig. 4. 


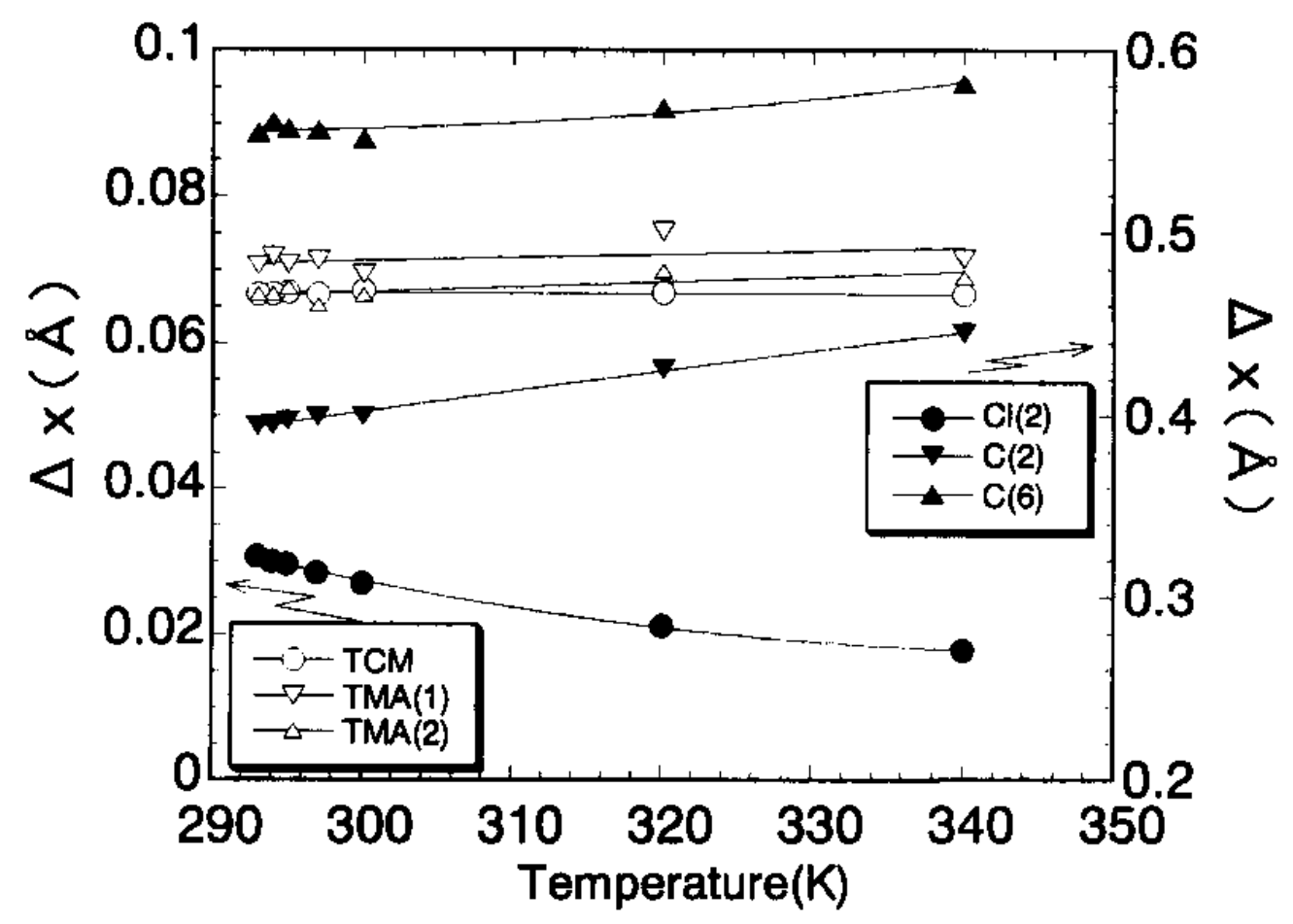

Fig. 5.

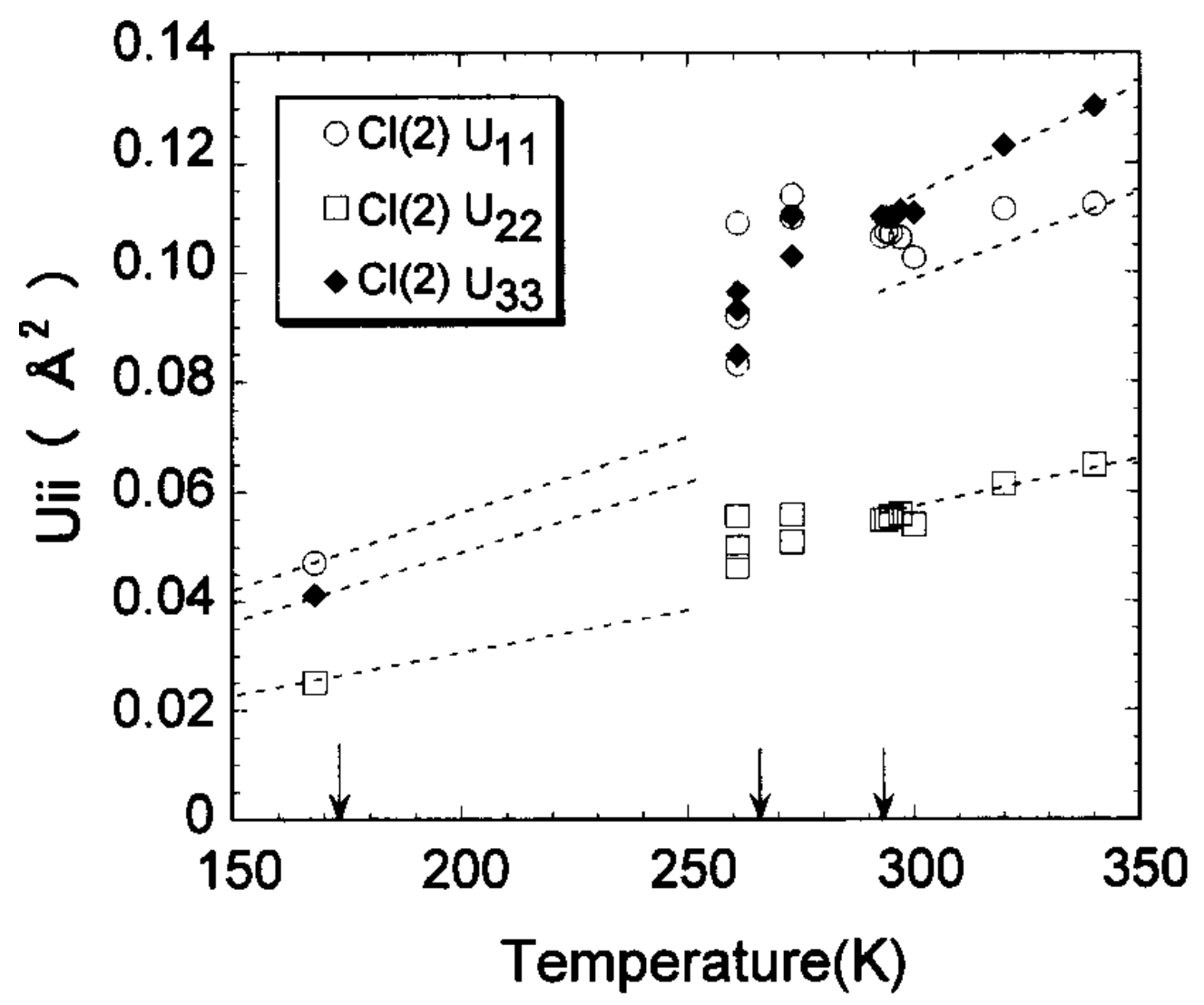

Fig. 6. 


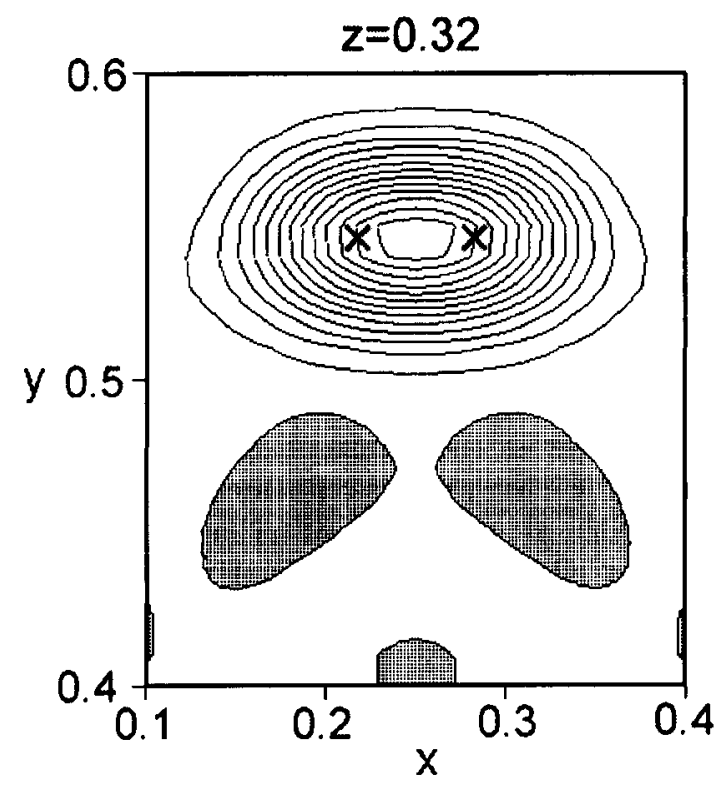

(a)

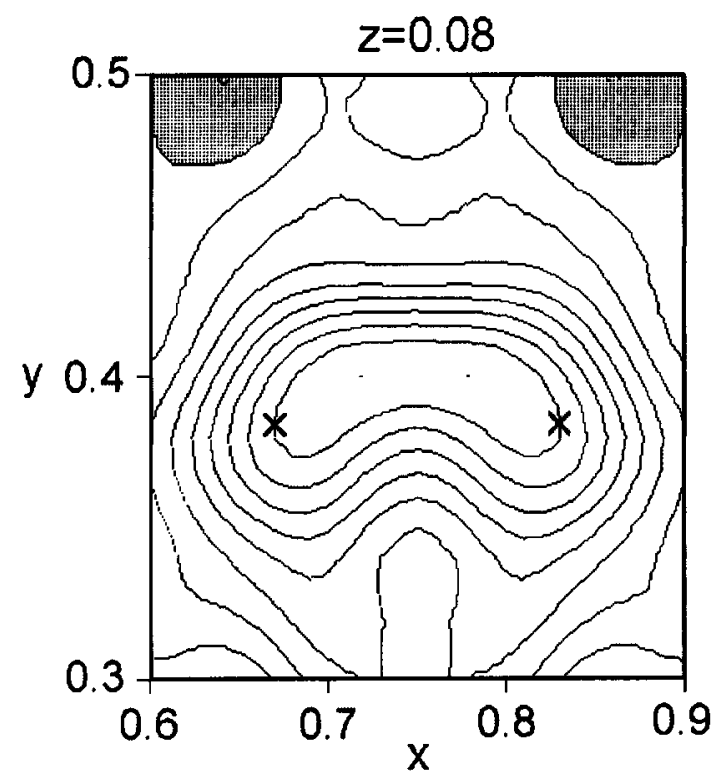

(b)

Fig. 7. 